\title{
Charge-Kondo effect mediated by repulsive interactions
}

\author{
S. Mojtaba Tabatabaei \\ Department of Physics, Shahid Beheshti University, Tehran, Iran
}

(Dated: June 20, 2018)

\begin{abstract}
We investigate the Kondo effect in a double-quantum-dot which is capacitively coupled to a charge-Qubit. It is shown that due to this capacitive coupling, the bare inter-dot repulsive interaction in the double-quantum-dot is effectively reduced and eventually changed to an attractive interaction for strong couplings between the double-quantum-dot and the Qubit. By deriving the low-energy effective Hamiltonian of the system, we find that the low energy dynamics of the system corresponding to these two positive or negative effective interaction regimes can be described, respectively, by an isotropic orbital-Kondo or an anisotropic charge-Kondo Hamiltonian. Moreover, we study various thermodynamic and electronic transport properties of the system by using the numerical renormalization group method.
\end{abstract}

\section{INTRODUCTION}

During last two decades, the Kondo effect in quantum dots(QD) has become an attractive research field in condensed matter physics 10. The main motivation for these studies is the possibility to explore various aspects of such an important effect in an experimentally accessible and fully tunable manner. Theoretically, Kondo effect is expected to be arisen at low temperatures whenever a localized system with a degenerate ground state is coupled to an environment with the same degeneracy and it is usually referred to the formation of a zero energy resonance state in the density of states of the system as a result of higher order tunneling processes between the localized system and the environment ${ }^{1}$. Depending on the nature of the degrees of freedom contributing to the manifestation of this effect, different kinds of Kondo effects have been identified in the literature. For example, the spin-Kondo effect is the most addressed Kondo effect which is associated with the fluctuations in the degenerate spin-up and down states in the local system 11 13. Another example is the orbital-Kondo effect which is formed in a double-quantum-dot(DQD) and associated with the degenerate pseudo-spin states corresponding to the occupation of the DQD by an electron in its left or right dot $14-16$.

The other somehow elusive Kondo effect is the chargeKondo effect which is associated with the fluctuations in degenerate states with different charge occupations. In this sense, the charge-Kondo effect is expected tooccur in negative- $U$ centers where an attractive interaction makes the doubly occupied or empty states have lower energies than the singly occupied states. Despite the fact that the theory of charge-Kondo effect was developed in early $1990 \sqrt{17}$, its experimental realization has not been reached until recent years. It was first reported in the bulk $P b T e$ semiconductors doped with $T l$ valence skipping elements which are in essence acting as negative- $U$ centers in the host material 21 . Another observation of the chargeKondo effect was also reported in transport through single electron transistors formed at the $\mathrm{LaAlO}_{3} / \mathrm{SrTiO}_{3}$ interfaces 22 24. Meanwhile, some efforts have been also devoted to engineer the attractive interaction required for the charge-Kondo effect by introducing other degrees of freedom interacting with electrons. Refs. 25 33] are examples of such theoretical proposals.

Recently, Hamo et.a ${ }^{34}$ reported the observation of attracting electrons in a setup composed of a carbon nanotube double quantum dot(DQD) along with a chargeQubit both of which are constructed on separate microchips, placed one above the other and perpendicular to each other. They found that an attractive interaction will be induced between the electrons in the DQD when the height of the Qubit above the DQD becomes less than a specific value. The attraction mechanism in their setup, as is discussed by Little ${ }^{35}$, is purely electronic and of excitonic origin: An electron on the DQD repels the electron in the up state of the Qubit leaving behind it a cloud of positive charge on the Qubit which in turn, this positive charge will attract the other electron on the DQD making the whole as two attracting electrons on the DQD.

In view of the presence of attractive electrons in the DQD-Qubit system, it is natural to ask whether the electronic transport through DQD could show the chargeKondo effect or not? It should be emphasized that in their experiment, Hamo et.al, observed a conductance enhancement on the degeneracy points between the two empty and doubly occupied states of the DQD, a signature which was assigned to the charge-Kondo effect by them. However, so far, there has not been presented a rigorous description for the Kondo effect in the DQDQubit coupled system in the literature.

In the present work, we theoretically explore the characteristics of the Kondo effect in the DQD-Qubit coupled system. In the rest of the paper, in Sec II we at first introduce the model Hamiltonian of the DQD-Qubit system and discuss the effective interaction induced in the DQD due to its coupling with the Qubit. Then, in Sec III] we derive the low-energy effective Hamiltonian of the system by using the Raleigh-Schrödinger degenerate perturbation theory 3036 . We show that for positive effective interaction in the DQD, the system exhibits isotropic orbital-Kondo effect while in the negative effective interaction regime, the anisotropic charge-Kondo effect will be manifested in the system at low enough temperatures. 


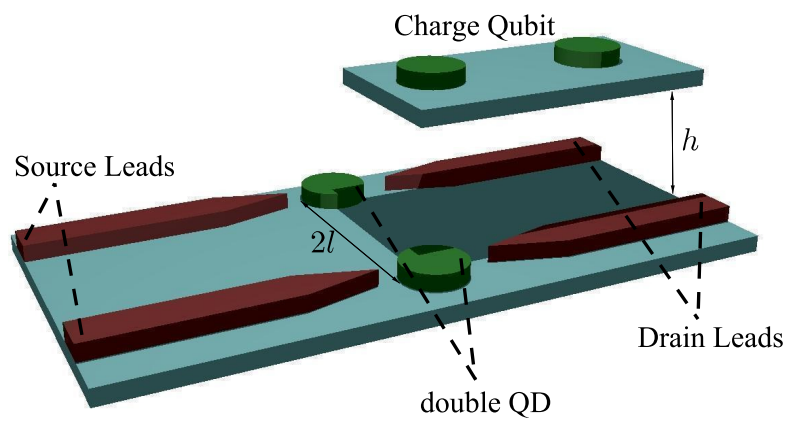

Figure 1. The charge Qubit is suspended above the DQD at height $h$ and midway between the two dots of the DQD. Each dot of the DQD is coupled to its own source and drain electrodes and the distance between them is $2 l$. One of the Qubit's dots has smaller distance to the DQD than the second Qubit's dot and therefore, the capacitive effect of the second dot of the Qubit on the dynamics of the DQD is overestimated.

Then in Sec IV, we supplement our study with the results obtained by using the numerical renoramlization group method(NRG $)^{37}$ to confirm the presence of these different Kondo effects in the system and extract some of the DQD's transport properties in the charge-Kondo regime. Finally, in Sec $\mathrm{V}$, we give the conclusions.

\section{THE MODEL}

Our model system which is shown in Fig 1 , consists of a parallel DQD and a charge Qubit. The Qubit is placed on a separate host than DQD and is positioned above the mid-point between DQD at height $h$, so that the DQD and the Qubit can interact with each other only in a repulsive manner. The DQD is constituted from two dots while each of them is contacted to its own source and drain electrodes. The total Hamiltonian of the system is given by

$$
\hat{\mathcal{H}}=\hat{\mathcal{H}}_{S}+\hat{\mathcal{H}}_{L}+\hat{\mathcal{H}}_{T} .
$$

The first term is the Hamiltonian of the DQD-Qubit system, which is given by $\hat{\mathcal{H}}_{S}=\hat{\mathcal{H}}_{D Q D}+\hat{\mathcal{H}}_{q u b i t}+\hat{\mathcal{H}}_{I}$. The Hamiltonian of the DQD is given by

$$
\hat{\mathcal{H}}_{D Q D}=\varepsilon_{1} \hat{n}_{1}+\varepsilon_{2} \hat{n}_{2}+U \hat{n}_{1} \hat{n}_{2},
$$

where $\hat{n}_{i}=\hat{d}_{i}^{\dagger} \hat{d}_{i}, \hat{d}_{i}^{\dagger}$ for $i=1,2$ creates an electron in the respective $\mathrm{QD}, \varepsilon_{i}$ is the applied gate voltage and $U$ is the inter-dot electron-electron interaction energy which is assumed to be a positive constant. In order to capture the main physics of the charge-Kondo effect, we consider the electrons to be spinless (by applying a large magnetic field) and therefore, each QD can be occupied only by a single electron. Moreover, $\hat{\mathcal{H}}_{\text {qubit }}$ denotes the Hamiltonian of the Qubit which is given by

$$
\hat{\mathcal{H}}_{\text {qubit }}=-\frac{\omega_{0}}{2} \hat{\tau}_{z}+\frac{\Delta}{2} \hat{\tau}_{x}
$$

where $\omega_{0}$ is the energy difference between the energy levels on either dots of the Qubit, $\Delta / 2$ gives the corresponding electron hybridization in the Qubit and $\hat{\tau}_{x}$ and $\hat{\tau}_{z}$ are the usual Pauli operators operating in the Qubit's Hilbert space and defined respectively by $\hat{\tau}_{x}=(|\Uparrow\rangle\langle\Downarrow|+| \Downarrow\rangle\langle\Uparrow|)$ and $\hat{\tau}_{z}=(|\uparrow\rangle\langle\Uparrow|-| \Downarrow\rangle\langle\Downarrow|)$, where $|\uparrow\rangle$ and $|\Downarrow\rangle$ represent the two charge sates of the Qubit. Furthermore, the interaction Hamiltonian of the DQD-Qubit is given by

$$
\hat{\mathcal{H}}_{I}=\frac{\lambda}{2} \hat{n}_{d} \hat{\tau}_{z}
$$

where $\hat{n}_{d}=\hat{n}_{1}+\hat{n}_{2}$ and $\lambda$ is the capacitive coupling energy between DQD and Qubit which is assumed to be a positive constant. The value of $\lambda$ is proportional to $r_{\lambda}^{-1}$, where $r_{\lambda}=\sqrt{l^{2}+h^{2}}$ is the distance between the charge Qubit and the DQD (see Fig, 1 for the definitions of $l$ and $h$ ).

The DQD is tunnel coupled to four normal metal electrodes so that each dot coupled to its own source and drain electrodes. The electrodes are described by $\hat{\mathcal{H}}_{L}=$ $\sum_{i, j} \hat{\mathcal{H}}_{L, i, j}$, where $j=S, D$ denote source and drain electrodes for the respective $\operatorname{dots} i=1,2$, and $\hat{\mathcal{H}}_{L, i, j}=$ $\sum_{k} \varepsilon_{k} \hat{c}_{k, i, j}^{\dagger} \hat{c}_{k, i, j}$, where $\hat{c}_{k, i, j}^{\dagger}$ is the corresponding operator for electron creation with energy $\varepsilon_{k}$ in the respective electrodes. The hybridization of each dot of the DQD with their electrodes is assumed to be energy independent and characterized by a hybridization constant $t$ and is described by $\hat{\mathcal{H}}_{T}=\sum_{k, i, j} t\left(\hat{c}_{k, i, j}^{\dagger} \hat{d}_{i}+h . c\right)$. The Hamiltonian of each source and drain electrode corresponding to each dot may be transformed to the Hamiltonian of a single lead by using the canonical transformations $\hat{\tilde{c}}_{k, i}=\left(\hat{c}_{k, i, S}+\hat{c}_{k, i, D}\right) / \sqrt{2}$ and $\hat{\tilde{b}}_{k, i}=\left(\hat{c}_{k, i, S}-\hat{c}_{k, i, D}\right) / \sqrt{2}$ and then, the expressions of the leads and the DQD-leads Hamiltonians are given, respectively, by

$$
\hat{\mathcal{H}}_{L}=\sum_{k, i} \varepsilon_{k} \hat{\tilde{c}}_{k, i}^{\dagger} \hat{\tilde{c}}_{k, i}
$$

and

$$
\hat{\mathcal{H}}_{T}=\sum_{k, i} t\left(\hat{\tilde{c}}_{k, i}^{\dagger} \hat{d}_{i}+h . c\right) .
$$

In order to learn more about the impact of the capacitive interaction between DQD and Qubit on the behavior of the system, it is useful to calculate the eigenstates and their energies of the isolated DQD-Qubit system. The eigenenergies of $\mathcal{H}_{S}$ are obtained straightforwardly from $\mathcal{H}_{S}\left|\psi_{ \pm}^{n_{1}, n_{2}}\right\rangle=E_{ \pm}^{n_{1}, n_{2}}\left|\psi_{ \pm}^{n_{1}, n_{2}}\right\rangle$, as

$$
E_{ \pm}^{n_{1}, n_{2}}=n_{1} \varepsilon_{1}+n_{2} \varepsilon_{2}+n_{1} n_{2} U \pm \frac{\Omega_{n_{d}}}{2}
$$

where $n_{d}=n_{1}+n_{2}$ is the total occupation of DQD and $\Omega_{n}=\sqrt{\Delta^{2}+\left(\omega_{0}-n \lambda\right)^{2}}$. Moreover, the eigenstates are found to be $\left|\psi_{ \pm}^{n_{1}, n_{2}}\right\rangle=\left|n_{1}, n_{2}\right\rangle_{D Q D}\left|n_{d}, \pm\right\rangle_{q u b i t}$, where $\left|n_{1}, n_{2}\right\rangle_{D Q D}$ is the occupation state of DQD and 
$\left|n_{d}, \pm\right\rangle_{\text {qubit }}$ is the Qubit eigenstate given by

$$
\left|n_{d}, \pm\right\rangle_{q u b i t}=b_{ \pm}^{n_{d}}\left(\left(-\omega_{0}+n_{d} \lambda \pm \Omega_{n_{d}}\right)|\Uparrow\rangle+\Delta|\Downarrow\rangle\right),
$$

where $b_{ \pm}^{n_{d}}$ is a normalization constant. Looking at the eigenenergies, Eq.(7), we can understand that as a direct consequence of the coupling with Qubit, the electronelectron interaction in DQD is reduced to an effective interaction equal to

$$
\begin{aligned}
U_{e f f} & =E_{-}^{1,1}+E_{-}^{0,0}-E_{-}^{1,0}-E_{-}^{0,1} \\
& =U+\Omega_{1}-\frac{1}{2}\left(\Omega_{2}+\Omega_{0}\right) .
\end{aligned}
$$

More interestingly, we see that when $U<\frac{1}{2}\left(\Omega_{2}+\Omega_{0}\right)-$ $\Omega_{1}$, the sign of $U_{\text {eff }}$ becomes negative which means that in such situations, there presents a net attractive interaction between the electrons in the DQD. Even though it may seem surprising at first glance, we can get some feelings of this attractive interaction by noting that this is indeed an induced attraction between the electrons. In other words, the presence of the oscillating polarization field of the Qubit on the electrons in the DQD, dresses their electric potential and forces them to favor the doubly occupied states more that the singly ones which in turn can be considered as such that the two electrons attract each other $34 \mid 35$

Another feature in Eq. (7) is that for some particular parameter configurations, the ground-state of the system becomes degenerate, composed of the two states $\left|\psi_{-}^{0,1}\right\rangle$ and $\left|\psi_{-}^{1,0}\right\rangle\left(\left|\psi_{-}^{0,0}\right\rangle\right.$ and $\left.\left|\psi_{-}^{1,1}\right\rangle\right)$ in $U_{\text {eff }}>0\left(U_{\text {eff }}<0\right)$ regime. Accordingly, we can expect that when the subsystem of DQD-Qubit with the degenerate ground-state is appropriately coupled to the electrodes, higher order electron tunneling processes between DQD and electrodes dress this degenerate state and form a many-body Kondo resonance and hence a Kondo effect arises in the system.

\section{LOW-ENERGY EFFECTIVE HAMILTONIAN}

In order to identify the characteristics of the Kondo effect in our model system, it is sufficient to derive a low-energy effective Hamiltonian describing the lowtemperature dynamics of the system up to the secondorder of the electron tunneling processes in the DQD. Here, we present the main results and relegate the technical details to the Appendix A. By using the projection operators

$$
\hat{P}_{ \pm}^{n_{1}, n_{2}}=\left|\psi_{ \pm}^{n_{1}, n_{2}}\right\rangle\left\langle\psi_{ \pm}^{n_{1}, n_{2}}\right|
$$

we can calculate the effective Hamiltonian of the system by using $30 \mid 36$

$$
\hat{\mathcal{H}}_{e f f}=\sum_{\substack{n_{1}, n_{2}=0,1 \\ \nu= \pm}} \frac{\hat{P}_{0} \hat{\mathcal{H}}_{T} \hat{P}_{\nu}^{n_{1}, n_{2}} \hat{\mathcal{H}}_{T} \hat{P}_{0}}{E_{0}-E_{\nu}^{n_{1}, n_{2}}},
$$

where $\hat{P}_{0}$ and $E_{0}$ are the corresponding projector operator and energy of the ground state of the unperturbed system, respectively.

In positive $U_{\text {eff }}$ regime, the ground-state of the system can become degenerate (which is essential for the Kondo effect to be arisen in the system) and constituted from the two states $\left|\psi_{-}^{0,1}\right\rangle$ and $\left|\psi_{-}^{1,0}\right\rangle$ when the conditions $\lambda=\omega_{0}, \varepsilon_{1}+\frac{U}{2}=\varepsilon_{2}+\frac{U}{2}=V_{g}$ and $\left|V_{g}\right|<U_{\text {eff }} / 2$, are satisfied. Then, using Eq.(11), the low-energy effective Hamiltonian can be obtained by

$$
\begin{aligned}
\hat{\mathcal{H}}_{e f f} & =\sum_{\substack{n_{1}, n_{2}=0,1 \\
\nu= \pm}} \frac{\left(\hat{P}_{-}^{1,0}+\hat{P}_{-}^{0,1}\right) \hat{\mathcal{H}}_{T} \hat{P}_{\nu}^{n_{1}, n_{2}} \hat{\mathcal{H}}_{T}\left(\hat{P}_{-}^{1,0}+\hat{P}_{-}^{0,1}\right)}{E_{-}^{1,0}-E_{\nu}^{n_{1}, n_{2}}} \\
& \approx J \vec{S}_{d} \cdot \vec{S}_{c},
\end{aligned}
$$

where $\vec{S}_{d}$ and $\vec{S}_{c}$ are the corresponding pseudo-spin vector of DQD and electrodes, respectively, which are defined in Eq. A7 and $J$ is the Kondo exchange coupling constant which is given by

$$
J=\frac{t^{2}}{\left(V_{g}+U\right)-\frac{(\lambda / 2)^{2}}{\Delta+V_{g}+U}}-\frac{t^{2}}{V_{g}-\frac{(\lambda / 2)^{2}}{V_{g}-\Delta}} .
$$

Thus, we see that the low-temperature dynamics of the system in $U_{\text {eff }}>0$ regime is governed by a $S U(2)$ isotropic orbital-Kondo Hamiltonian in which a single electron on DQD plays the role of a pseudo-spin making a singlet state with the corresponding pseudo spins of the electrodes. The Kondo temperature which is the particular temperature below which the Kondo effect is manifested in the system, is given in this case by

$$
T_{K}^{i}=\alpha \exp \left[-\frac{1}{\rho_{0} J}\right]
$$

where $\alpha$ is a proportionality constant and $\rho_{0}=1 /(2 D)$ is the density of states of the leads which is assumed to be constant in the wide-band approximation and $D$ is the half band width of the electrodes. To the lowest order in $\lambda$, the correction to the value of $J$ equals to

$$
\begin{gathered}
J \approx-\frac{t^{2} U}{V_{g}\left(V_{g}+U\right)}+\frac{t^{2}(\lambda / 2)^{2}}{\left(V_{g}+U\right)^{2}\left(\Delta+V_{g}+U\right)}+ \\
\frac{t^{2}(\lambda / 2)^{2}}{V_{g}^{2}\left(\Delta-V_{g}\right)}+\mathcal{O}\left(\lambda^{4}\right) .
\end{gathered}
$$

Hence, in the positive $U_{\text {eff }}$ regime, the DQD-Qubit coupling results in an enhancement of the Kondo temperature of the system. Note that for $\lambda=0$, Eq. 13 correctly reproduces the results of the conventional orbital-Kondo effect in a DQD.

For negative $U_{\text {eff }}$ regime, by demanding the groundstate to be degenerate and constituted from the two states $\left|\psi_{-}^{0,0}\right\rangle$ and $\left|\psi_{-}^{1,1}\right\rangle$, it can be found that the the sufficient conditions are $\lambda=\omega_{0}, \varepsilon_{1}+\varepsilon_{2}+U=0$ and $\left|V_{z}\right|<-U_{\text {eff }} / 2$, where $V_{z}=\varepsilon_{1}+U / 2=-\left(\varepsilon_{2}+U / 2\right)$. 
Now, we can perform a second-order perturbation to obtain the low-energy effective Hamiltonian of the system as

$$
\begin{aligned}
\hat{\mathcal{H}}_{e f f} & =\sum_{\substack{n_{1}, n_{2}=0,1 \\
\nu= \pm}} \frac{\left(\hat{P}_{-}^{0,0}+\hat{P}_{-}^{1,1}\right) \hat{\mathcal{H}}_{T} \hat{P}_{\nu}^{n_{1}, n_{2}} \hat{\mathcal{H}}_{T}\left(\hat{P}_{-}^{0,0}+\hat{P}_{-}^{1,1}\right)}{E_{-}^{0,0}-E_{\nu}^{n_{1}, n_{2}}} \\
& \approx\left(J_{\|} I_{d}^{z} I_{c}^{z}+J_{\perp}\left(I_{d}^{x} I_{c}^{x}+I_{d}^{y} I_{c}^{y}\right)\right),
\end{aligned}
$$

where $\vec{I}_{d}\left(\vec{I}_{c}\right)$ are the corresponding iso-spin operators of the DQD(electrodes) which are defined by applying a special particle-hole transformation, i.e $d_{2}^{\dagger} \rightarrow d_{2}$ and $\tilde{c}_{k, 2}^{\dagger} \rightarrow-\tilde{c}_{k, 2}$, on the $\vec{S}_{d}\left(\vec{S}_{c}\right)$ operators and are given in Eq. A10. Furthermore, the two parallel and transverse exchange Kondo coupling constants $J_{\|}$and $J_{\perp}$ are given by

$$
\begin{aligned}
& J_{\|}=2 t^{2}\left[\frac{-\lambda+U+2 V_{z}-\frac{\Delta^{2}\left(\lambda+2 \Omega_{0}\right)}{\Delta^{2}+\lambda^{2}+\lambda \Omega_{0}}}{\left(U+2 V_{z}-\Omega_{0}\right)^{2}-\Delta^{2}}\right. \\
& \left.-\frac{\lambda-U+2 V_{z}+\frac{\Delta^{2}\left(\lambda+2 \Omega_{0}\right)}{\Delta^{2}+\lambda^{2}+\lambda \Omega_{0}}}{\left(-U+2 V_{z}+\Omega_{0}\right)^{2}-\Delta^{2}}\right], \\
& J_{\perp}=\frac{2 \Delta t^{2}}{\Omega_{0}}\left(\frac{1}{2 V_{z}-U+\frac{\lambda^{2}}{2 V_{z}-U+2 \Omega_{0}}}-\right. \\
& \left.\frac{1}{2 V_{z}+U+\frac{\lambda^{2}}{2 V_{z}+U-2 \Omega_{0}}}\right) \text {. }
\end{aligned}
$$

Thus, the low-energy dynamics of the DQD-Qubit system in the $U_{e f f}<0$ regime is governed by an anisotropic charge-Kondo Hamiltonian. In this case, the Kondo temperature is given by

$$
T_{K}^{a}=\beta\left(\frac{J_{\|}+\sqrt{J_{\|}^{2}-J_{\perp}^{2}}}{J_{\|}-\sqrt{J_{\|}^{2}-J_{\perp}^{2}}}\right)^{-\frac{1}{4 \rho_{0} \sqrt{J_{\|}^{2}-J_{\perp}^{2}}}},
$$

where $\beta$ is a proportionality constant.

Before proceeding to present our numerical results, we note that according to Eqs. (13) and (17), it seems that a quantum phase transition to ferromagnetic phase is possible in the effective Hamailtonian of the system. However, it should be noticed also that by choosing the appropriate parameters regime for deriving the effective Hamiltonian in the above calculations, the system is certainly in the Kondo regime and could not cross over to a ferromagnetic phase within their corresponding parameters regime ${ }^{32}$.

\section{NUMERICAL RENORMALIZATION GROUP RESULTS}

Here, we provide numerical results obtained by numerical renormalization group(NRG) method to confirm the manifestation of the Kondo effect in the DQD-Qubit system. The NRG results are obtained using the "NRG
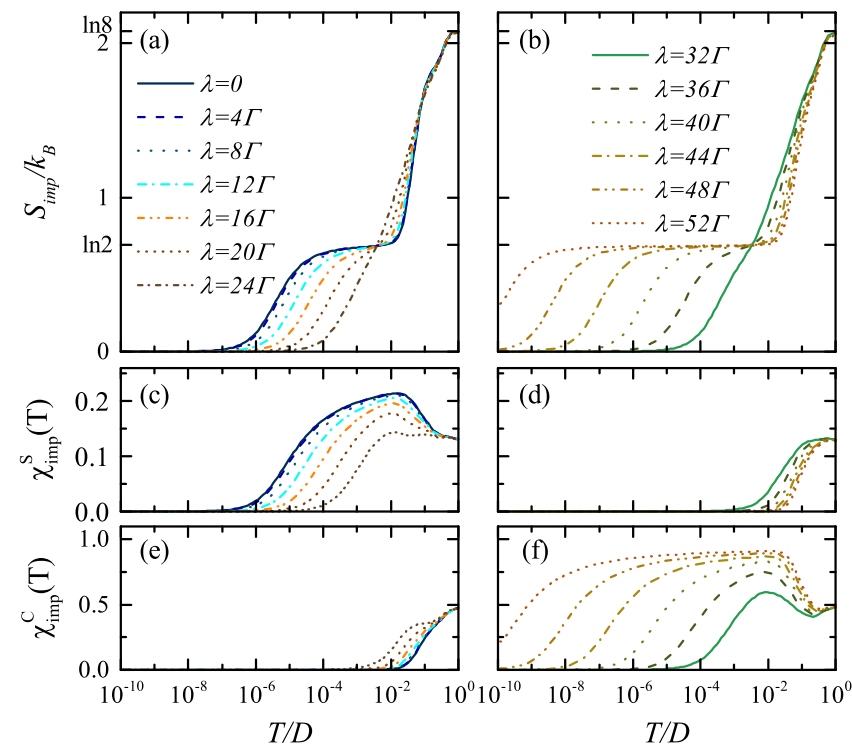

Figure 2. Impurity contribution to the total entropy(top panels), magnetic susceptibility(middle panels) and charge susceptibility(bottom panels) of the system. The values of $\lambda$ in the left and right panels are corresponding to the positive and negative $U_{\text {eff }}$ regimes, respectively. Other parameters are $\varepsilon_{1}=\varepsilon_{2}=-U / 2, U=20 \Gamma, \omega_{0}=\lambda$ and $\Delta=10 \Gamma$.

LJUBLJANA" package $\mathrm{e}^{38}$ by solving the total Hamiltonian in Eq. (1) with $\Gamma=\pi \rho_{0} t^{2}=0.01 D$, where $\rho_{0}=1 /(2 D)$ is the density of states of the leads which is assumed to be constant in the wide-band approximation and $D=1$ is taken as the unit of the energy scales. Moreover, we take $U=20 \Gamma$ and $\Delta=10 \Gamma$. We mention that for the chosen values of $U$ and $\Delta$, the particular value of $\lambda$ at which $U_{\text {eff }}$ will be vanished is $\lambda \approx 28 \Gamma$. Thus, the system is expected to be in positive (negative) $U_{\text {eff }}$ regime when $\lambda<28 \Gamma(\lambda>28 \Gamma)$.

First, we investigate the thermodynamic properties of the system. In Fig 2, we show the temperature dependence of the impurity contribution to the entropy $S_{i m p}(T)$, orbital pseudo-spin magnetic susceptibility $\left.\chi_{i m p}^{S}(T)=\left(\left\langle\left(S^{z}\right)^{2}\right\rangle-\left\langle\left(S^{z}\right)^{2}\right\rangle_{0}\right) / T\right)$ where $S^{z}=$ $S_{d}^{z}+S_{c}^{z}$ and $\langle\ldots\rangle_{0}$ denotes the thermal expectation value in the absence of the DQD and Qubit, and the total charge susceptibility $\chi_{i m p}^{C}(T)=\left(\left\langle Q^{2}\right\rangle-\left\langle Q^{2}\right\rangle_{0}\right) / T$ of the system in the $\mathrm{p}-\mathrm{h}$ symmetric point and for several $\lambda$ values corresponding to the both $U_{\text {eff }}>0$ and $U_{\text {eff }}<0$ regimes. As it is seen in Figs 2(a) and (b), at high temperatures, the system is in its free orbital fixed point where all $2^{3}$ states of the DQD-Qubit system are equally probable and hence, the entropy becomes $S_{i m p}=k_{B} \ln 8$. By decreasing the temperature, the system crosses over to a local moment fixed point with $S_{i m p}=k_{B} \ln 2$ which means the presence of only two degrees of freedom in the system. The nature of this local moment could be revealed by looking at the respective magnetic and charge susceptibilities of the system which are shown in Figs 2(c)-(f). It is seen that, the local moment in the 

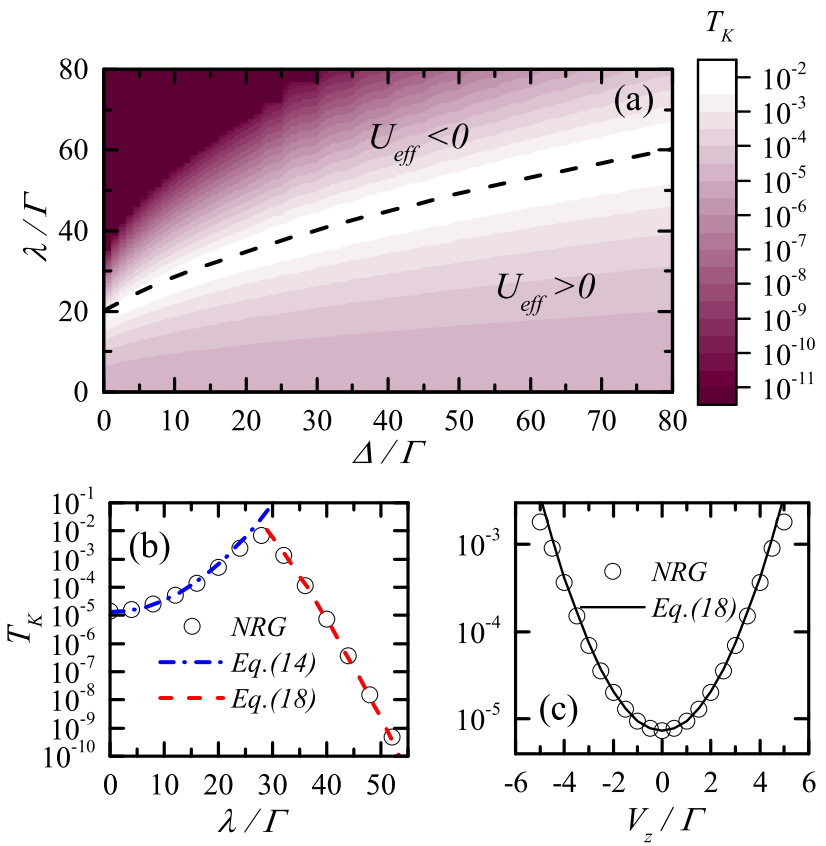

Figure 3. (a) Kondo temperature of the system calculated from NRG results with respect to the $\lambda$ and $\Delta$. Dashed line shows the boundary between positive and negative $U_{\text {eff }}$ regimes. (b) Comparison of Kondo temperature of the system for $\Delta=10 \Gamma$, calculated by NRG(circles), Eq. 14)(dashdotted line) and Eq. 18 (dashed line). (c) Kondo temperature of the system as a function of $V_{z}$, calculated by NRG(circles), Eq. 18 (dashed line), for $\Delta=10 \Gamma, \lambda=40 \Gamma$. The proportionality constants in Eqs. (14) and 18 are calculated by numerical fitting to be $\alpha=0.034$ and $\beta=0.016$. Other parameters are $\varepsilon_{1}=\varepsilon_{2}=-U / 2, U=20 \Gamma, \omega_{0}=\lambda$.

$U_{\text {eff }}>0$ regime is accompanied by formation of an orbital pseudo-spin magnetic moment in the system (see Fig 2(c)). On the other hand, in the $U_{\text {eff }}<0$ regime, we see that the established local moment is of charge type (see Fig 2(f)). Further decrease of the temperature results in fully screening this local moment after which the system crosses over to its Kondo strong coupling fixed point with $S_{i m p}=0$.

The particular temperature at which the local moment of the system quenches in Figs.2(a) and (b) is usually called the Kondo temperature of the system. In Fig 3 (a), we have shown $T_{K}$ values of the system calculated by the relation $S_{i m p}\left(T_{K}\right)=0.5 k_{B} \ln 2$ from NRG results. The dashed line shows the points on which the values of $U_{\text {eff }}$ vanish. It is seen that the behavior of $T_{K}$ values in the positive $U_{\text {eff }}$ region differs from that in negative $U_{\text {eff }}$ region. This is more obvious in Fig, $3(\mathrm{~b})$, where we have plotted the details of Fig 3 (a) for a particular value of $\Delta$. As we anticipated in Sec III in the positive $U_{\text {eff }}$ region, increasing $\lambda$ results in an increase of the $T_{K}$ values, while in the negative $U_{\text {eff }}$ regime, the value of $T_{K}$ is drastically decreased by increasing $\lambda$. In Fig $3(\mathrm{~b})$, we have also compared the NRG results with the results obtained using Eqs.(14) and (18), where we

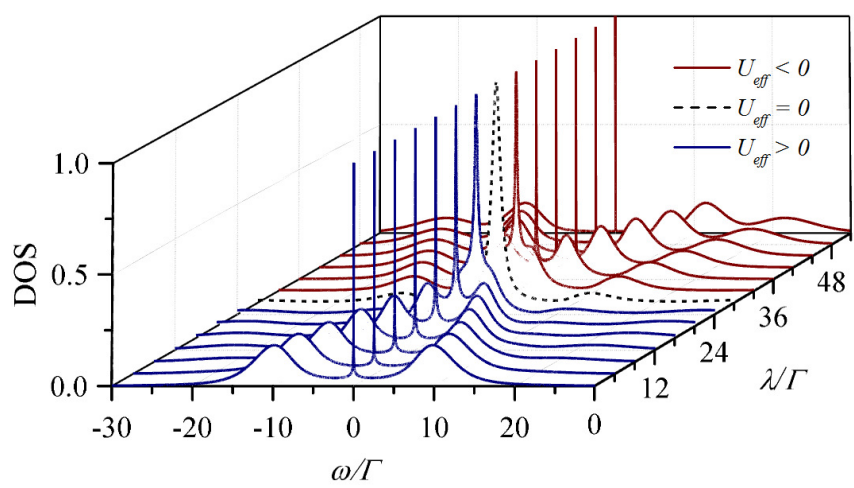

Figure 4. The spectral function(DOS) of the DQD for $\lambda$ values ranging from $\lambda=0$ (orbital-Kondo regime) to $\lambda=52 \Gamma$ (chargeKondo regime). Dashed line shows the DOS for the particular $\lambda$ value corresponding to the vanishing $U_{\text {eff }}$. Other parameters are $\varepsilon_{1}=\varepsilon_{2}=-U / 2, U=20 \Gamma, \omega_{0}=\lambda, \Delta=10 \Gamma$.

can see very good agreement between the two results. To complete our discussion about the Kondo temperature, in Fig 3(c), we show the dependence of $T_{K}$ on the values of $V_{z}$ where $V_{z}=\left(\varepsilon_{1}-\varepsilon_{2}\right) / 2$, and by assuming that the system is in the charge-Kondo regime. It is apparent that departures from $V_{z}=0$ give rise to an enhancement of $T_{K}$ which is again in agreement with the results obtained using Eq. 18.

Next, we consider spectral and transport properties of the DQD. In Fig 4, we show the spectral function of the DQD for different values of $\lambda$. As it is obvious, when the system is in orbital-Kondo regime, $\lambda<24 \Gamma$, there is a central Kondo peak along with two sideband peaks which are placed at the energies corresponding to the single particle excitation energies of DQD. By increasing $\lambda$, the system slightly crosses over to the chargeKondo regime which reflects in the central peak of the spectral function by first transforming it to a lorentzian form around $\lambda \approx 28 \Gamma$ and then to a sharp charge-Kondo peak for $\lambda>32 \Gamma$. Changing $\lambda$ has also affected not only the energies but also the numbers of the sideband peaks. This behavior can be explained by considering the single particle excitation energies of the isolated DQDQubit which could be calculated by using Eq. (7). In the orbital-Kondo regime, the ground state is the singly occupied states. Thus, there are four possible sideband peaks in this regime with energies equal to $\pm\left|E_{-}^{1,0}-E_{-}^{0,0}\right|$ and $\pm\left|E_{-}^{1,0}-E_{+}^{0,0}\right|$, which are associated with the single particle excitation in DQD without and with excitation of Qubit, respectively. However, as we can see in Fig, 4 , in orbital-Kondo regime, there are no sideband peaks with Qubit excitation in the spectral function which is mainly due to the weakness of the DQDQubit coupling. For larger $\lambda$ values, and specifically in the charge-Kondo regime, the sideband peaks with Qubit excitation are much more visible. We note that in the charge-Kondo regime, the ground state is composed of 

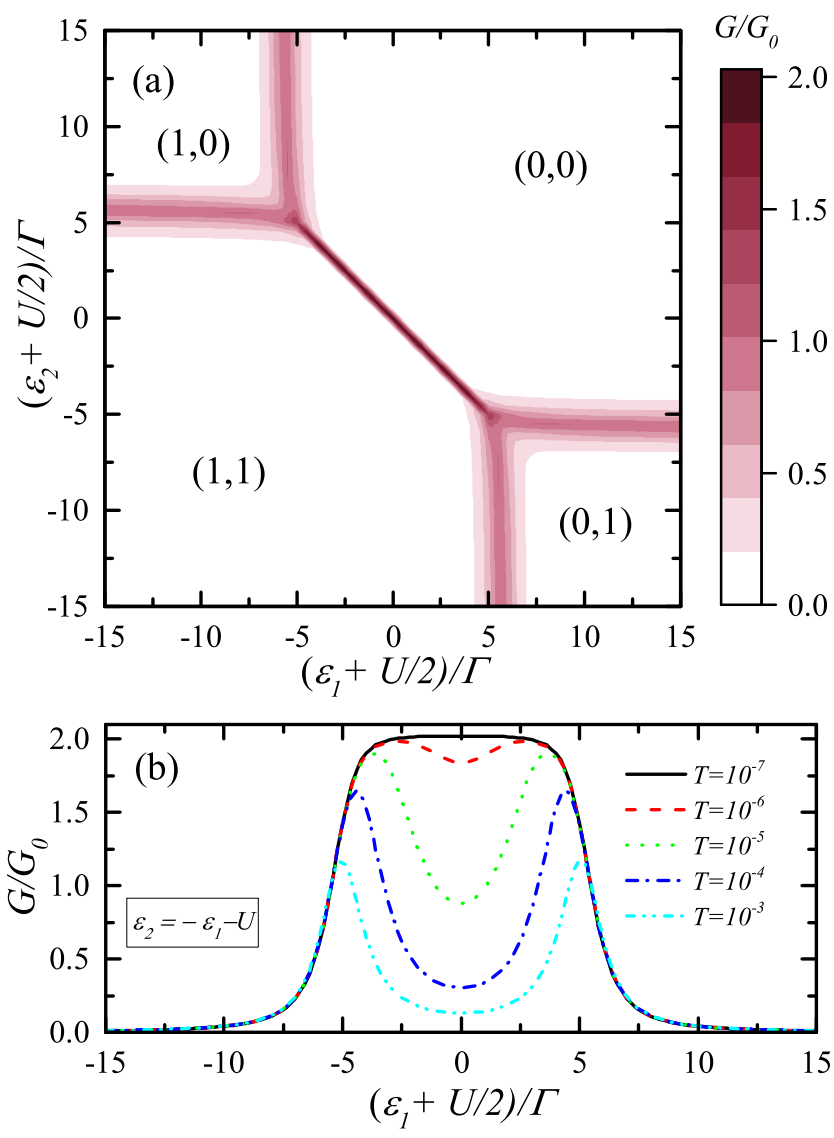

Figure 5. (a) Total linear conductance of the DQD with respect to the $\varepsilon_{1}$ and $\varepsilon_{2}$ values. Charge configurations of DQD are shown by $\left(n_{1}, n_{2}\right)$ on the figure. (b) Temperature dependence of the total linear conductance of DQD along the diagonal line in (a). Other parameters are $U=20 \Gamma, \Delta=$ $10 \Gamma, \omega_{0}=\lambda$ and $\lambda=40 \Gamma$.

the empty and doubly occupied states and therefore, the sideband peaks energies are obtained from $\pm\left|E_{-}^{0,0}-E_{-}^{1,0}\right|$ and $\pm\left|E_{-}^{0,0}-E_{+}^{1,0}\right|$.

In Fig 5 (a), we show the total conductance of DQD $(G)$ in the $\varepsilon_{1}-\varepsilon_{2}$ plane. The parameters are chosen such that the system is in the $U_{\text {eff }}<0$ regime. We see that the profile of the total conductance is rotated with respect to the usual charge stability diagrams expected for an interacting parallel $\mathrm{DQD}^{39}$. In particular, the presence of a long degeneracy between the two charge configurations $(0,0)$ and $(1,1)$ is a clue of an attractive interaction in the DQD. We can also infer the strength of this attractive interaction as the length of this degeneracy line segment between the two $(0,0)$ and $(1,1)$ regions. We have shown the temperature dependence of $G$ along this segment in Fig 5 (b) in which we can see that the conductance on this segment reaches the unitary value $G=2 G_{0}=2 e^{2} / h$ for enough low temperatures while for higher temperatures, it is suppressed except at the end points of the degeneracy segment. Hence, from these observations, we can deduce that the nature of this unitary conductance is of
charge-Kondo type. It should be emphasized that a similar conductance enhancement was reported in Ref. 34].

\section{CONCLUSIONS}

In conclusion, we considered a double-quantum-dot which is capacitively coupled to a charge Qubit. It is shown that this capacitive coupling renormalizes the inter-dot repulsive interaction in the double-quantum-dot and in some situations makes it to be an attractive interaction between the electrons in the double-quantumdot. We found that appropriate coupling of the doublequantum-dot with the electrodes could give rise to two different types of the Kondo effect depending on the sign of the inter-dot interaction in the double-quantum-dot. Namely, in the positive interaction regime, the system shows an isotropic orbital-Kondo effect while, in the negative interaction regime, an anisotropic charge-Kondo effect is expected to be shown in the system. By deriving the low-energy effective Hamiltonian of the system, we obtained the corresponding Kondo exchange coupling constants as well as the characteristic Kondo temperature of the system corresponding to these two different regimes. Moreover, we employed the numerical renormalization group method to confirm our analytical results and to extract some thermodynamic and electronic transport properties of the system.

\section{ACKNOWLEDGMENTS}

We would like to thank Farshad Ebrahimi for bringing the importance of the model system to our attention. We are also grateful to Pablo S. Cornaglia for useful comments and critical reading of a previous version of the manuscript and Rok Žitko for helpful comments.

\section{Appendix A: Derivation of effective Hamiltonian}

The low-energy effective Hamiltonian of the system can be calculated using the method of degenerate perturbation theory. We will calculate the second-order corrections to the unperturbed subsystem $\hat{\mathcal{H}}_{0}=\hat{\mathcal{H}}_{S}+\hat{\mathcal{H}}_{L}$ due to the perturbation coming from electron transitions between DQD and electrodes which is described by $\hat{\mathcal{H}}_{T}$. First, for future reference, we define eight projection operators to the subspace of the eigenstates $\left|\psi_{ \pm}^{n_{1}, n_{2}}\right\rangle$ by

$$
\begin{aligned}
& \hat{P}_{ \pm}^{0,0}=\left(1-\hat{n}_{1}\right)\left(1-\hat{n}_{2}\right)|0, \pm\rangle\langle 0, \pm|, \\
& \hat{P}_{ \pm}^{1,0}=\hat{n}_{1}\left(1-\hat{n}_{2}\right)|1, \pm\rangle\langle 1, \pm|, \\
& \hat{P}_{ \pm}^{0,1}=\left(1-\hat{n}_{1}\right) \hat{n}_{2}|1, \pm\rangle\langle 1, \pm|, \\
& \hat{P}_{ \pm}^{1,1}=\hat{n}_{1} \hat{n}_{2}|2, \pm\rangle\langle 2, \pm| .
\end{aligned}
$$


Now, the correction to the $\hat{\mathcal{H}}_{0}$ to the lowest order in $\hat{\mathcal{H}}_{T}$ can be calculated by

$$
\hat{\mathcal{H}}_{\text {eff }}=\sum_{\substack{n_{1}, n_{2}=0,1 \\ \nu= \pm}} \frac{\hat{P}_{0} \hat{\mathcal{H}}_{T} \hat{P}_{\nu}^{n_{1}, n_{2}} \hat{\mathcal{H}}_{T} \hat{P}_{0}}{E_{0}-E_{\nu}^{n_{1}, n_{2}}},
$$

where $\hat{P}_{0}$ denotes the projection to the specific groundstate of the unperturbed Hamiltonian $\hat{\mathcal{H}}_{0}$ with energy $E_{0}$. Because we are interested in deriving the Kondo Hamiltonian we restrict our calculations to the degenerate ground-states in the following discussions.

\section{Positive $U_{\text {eff }}$ regime}

In this regime, the ground state becomes degenerate when $E_{-}^{1,0}=E_{-}^{0,1}, E_{-}^{1,0}<E_{-}^{0,0}$ and $E_{-}^{1,0}<E_{-}^{1,1}$. Due to the vast parameters in the system a large number of possible parameter configurations can fulfill these conditions. By fixing $\lambda=\omega_{0}$, which makes the Qubit to be particlehole symmetric, we find that the necessary conditions for degeneracy in the ground-state become $\varepsilon_{1}=\varepsilon_{2}=V_{g}$ and $\left|\varepsilon_{1}+\frac{U}{2}\right|<\frac{1}{2}\left(U+\Omega_{1}-\Omega_{0}\right)$, where we have allowed for a gate voltage $V_{g}$ to consider the general case. By denoting the ground-state projector as $\hat{P}_{0}=\hat{P}_{-}^{1,0}+\hat{P}_{-}^{0,1}$, we can evaluate the effective Hamiltonian in this case by

$$
\hat{\mathcal{H}}_{e f f}=\sum_{\substack{n_{1}, n_{2}=0,1 \\ \nu= \pm}} \frac{\left\langle 1,-\left|\left(\hat{P}_{-}^{1,0}+\hat{P}_{-}^{0,1}\right) \hat{\mathcal{H}}_{T} \hat{P}_{\nu}^{n_{1}, n_{2}} \hat{\mathcal{H}}_{T}\left(\hat{P}_{-}^{1,0}+\hat{P}_{-}^{0,1}\right)\right| 1,-\right\rangle}{E_{-}^{1,0}-E_{\nu}^{n_{1}, n_{2}}} .
$$

Note that we have traced out the Qubit's degrees of freedom in order to obtain a pure electronic effective Hamiltonian. Explicit calculation of different terms in the above relation leads to

$$
\begin{gathered}
\sum_{\nu= \pm} \frac{\left\langle 1,-\left|\left(\hat{P}_{-}^{1,0}+\hat{P}_{-}^{0,1}\right) \hat{\mathcal{H}}_{T} \hat{P}_{\nu}^{0,0} \hat{\mathcal{H}}_{T}\left(\hat{P}_{-}^{1,0}+\hat{P}_{-}^{0,1}\right)\right| 1,-\right\rangle}{E_{-}^{1,0}-E_{\nu}^{0,0}}=\frac{4 t^{2}\left(-\Delta+V_{g}\right)}{4 V_{g}\left(\Delta-V_{g}\right)+\lambda^{2}} \times \\
\sum_{i=1,2}\left[-\delta_{q, p}\left(\hat{n}_{i}-\hat{n}_{1} \hat{n}_{2}\right)+\hat{\tilde{c}}_{q, i}^{\dagger} \hat{\tilde{c}}_{p, i} \hat{n}_{i}+\hat{\tilde{c}}_{q, i}^{\dagger} \hat{\tilde{c}}_{p, i} \hat{d}_{\bar{i}}^{\dagger} \hat{d}_{i}-\hat{\tilde{c}}_{q, i}^{\dagger} \hat{\tilde{c}}_{p, i} \hat{n}_{1} \hat{n}_{2}\right] \\
\sum_{\nu= \pm} \frac{\left\langle 1,-\left|\left(\hat{P}_{-}^{1,0}+\hat{P}_{-}^{0,1}\right) \hat{\mathcal{H}}_{T} \hat{P}_{\nu}^{1,1} \hat{\mathcal{H}}_{T}\left(\hat{P}_{-}^{1,0}+\hat{P}_{-}^{0,1}\right)\right| 1,-\right\rangle}{E_{-}^{1,0}-E_{\nu}^{1,1}}=\frac{4 t^{2}\left(\Delta+V_{g}+U\right)}{4\left(V_{g}+U\right)\left(\Delta+V_{g}+U\right)-\lambda^{2}} \times \\
\sum_{i=1,2}\left[-\hat{\tilde{c}}_{q, i}^{\dagger} \hat{\tilde{c}}_{p, i} \hat{n}_{\bar{i}}+\hat{\tilde{c}}_{q, i}^{\dagger} \hat{\tilde{c}}_{p, i} \hat{d}_{\bar{i}}^{\dagger} \hat{d}_{i}+\hat{\tilde{c}}_{q, i}^{\dagger} \hat{\tilde{c}}_{p, i} \hat{n}_{1} \hat{n}_{2}\right] .
\end{gathered}
$$

Summing the above two contributions to the effective Hamiltonian and using the identities $\hat{n}_{1} \hat{n}_{2}=1-\hat{n}_{d}$ and $\hat{n}_{d}=1$ which are valid only in the ground-state subspace of the positive $U_{\text {eff }}$ regime, we can reach to the following expression for $\hat{\mathcal{H}}_{e f f}$

$$
\hat{\mathcal{H}}_{e f f}=J \vec{S}_{d} \cdot \vec{S}_{c}+K \sum_{\substack{i, j=1,2 \\ q, p}} \hat{\tilde{c}}_{q, i}^{\dagger} \hat{\tilde{c}}_{p, i} / 2
$$

where $J$ and $K$ are given by

$$
\begin{array}{r}
J=\frac{t^{2}}{\left(V_{g}+U\right)-\frac{(\lambda / 2)^{2}}{\Delta+V_{g}+U}}-\frac{t^{2}}{V_{g}-\frac{(\lambda / 2)^{2}}{V_{g}-\Delta}}, \\
K=\frac{t^{2}}{\left(V_{g}+U\right)-\frac{(\lambda / 2)^{2}}{\Delta+V_{g}+U}}+\frac{t^{2}}{V_{g}-\frac{(\lambda / 2)^{2}}{V_{g}-\Delta}} .
\end{array}
$$


Moreover, $\vec{S}_{d}=\frac{1}{2} \sum_{i, j=1,2} \hat{d}_{i}^{\dagger} \vec{\sigma}_{i j} \hat{d}_{j}$ is the corresponding pseudo-spin vector of DQD which is represented by

$$
\begin{gathered}
S_{d}^{x}=\frac{1}{2}\left(\hat{d}_{2}^{\dagger} \hat{d}_{1}+\hat{d}_{2}^{\dagger} \hat{d}_{1}\right), \\
S_{d}^{y}=\frac{i}{2}\left(\hat{d}_{2}^{\dagger} \hat{d}_{1}-\hat{d}_{2}^{\dagger} \hat{d}_{1}\right), \\
S_{d}^{z}=\frac{1}{2}\left(\hat{n}_{1}-\hat{n}_{2}\right),
\end{gathered}
$$

and, analogously, $\vec{S}_{c}=\frac{1}{2} \sum_{i, j=1,2} \sum_{p, q} \hat{\tilde{c}}_{q, i}^{\dagger} \vec{\sigma}_{i j} \hat{\tilde{c}}_{p, j}$ is the pseudo-spin vector for the electrodes.

\section{Negative $U_{\text {eff }}$ regime}

In this regime, a similar calculation as that of positive $U_{\text {eff }}$ regime but for the the ground state composed of the two states $\left|\psi_{-}^{0,0}\right\rangle$ and $\left|\psi_{-}^{1,1}\right\rangle$ leads to the conditions $\lambda=\omega_{0}, \varepsilon_{1}+\varepsilon_{2}+U=0$ and $\left|V_{z}\right|<\frac{1}{2}\left(-U-\Omega_{1}+\Omega_{0}\right)$, where $V_{z}=\varepsilon_{1}+U / 2=-\left(\varepsilon_{2}+U / 2\right)$. Then, the ground-state projector is $\hat{P}_{0}=\hat{P}_{-}^{0,0}+\hat{P}_{-}^{1,1}$, and the effective Hamiltonian can be calculated by

$$
\hat{\mathcal{H}}_{e f f}=\sum_{\substack{n_{1}, n_{2}=0,1 \\ \nu= \pm}} \frac{\left(\left\langle0,-\left|\hat{P}_{-}^{0,0}+\langle 2,-| \hat{P}_{-}^{1,1}\right) \hat{\mathcal{H}}_{T} \hat{P}_{\nu}^{n_{1}, n_{2}} \hat{\mathcal{H}}_{T}\left(\hat{P}_{-}^{0,0}|0,-\rangle+\hat{P}_{-}^{1,1}|2,-\rangle\right)\right.\right.}{E_{-}^{0,0}-E_{\nu}^{n_{1}, n_{2}}} .
$$

By calculating the summations in the above relation, after some algebra, we get

$$
\begin{aligned}
& \sum_{\nu= \pm} \frac{\left\langle 0,-\left|\hat{P}_{-}^{0,0} \hat{\mathcal{H}}_{T} \hat{P}_{\nu}^{1,0} \hat{\mathcal{H}}_{T} \hat{P}_{-}^{0,0}\right| 0,-\right\rangle}{E_{-}^{0,0}-E_{\nu}^{1,0}}+\frac{\left\langle 2,-\left|\hat{P}_{-}^{1,1} \hat{\mathcal{H}}_{T} \hat{P}_{\nu}^{1,0} \hat{\mathcal{H}}_{T} \hat{P}_{-}^{1,1}\right| 2,-\right\rangle}{E_{-}^{0,0}-E_{\nu}^{1,0}}= \\
& \frac{2 t^{2}}{\Delta^{2}-\left(-U+2 V_{z}+\Omega_{0}\right)^{2}}\left(\lambda-U+2 V_{z}+\frac{\Delta^{2}\left(\lambda+2 \Omega_{0}\right)}{\left(\Delta^{2}+\lambda^{2}+\lambda \Omega_{0}\right)}\right) \times \\
& {\left[\hat{\tilde{c}}_{q, 1}^{\dagger} \hat{\tilde{c}}_{p, 1}-\hat{\tilde{c}}_{q, 1}^{\dagger} \hat{\tilde{c}}_{p, 1} \hat{n}_{d}+\delta_{q, p} \hat{n}_{1} \hat{n}_{2}+\left(\hat{\tilde{c}}_{q, 1}^{\dagger} \hat{\tilde{c}}_{p, 1}-\hat{\tilde{c}}_{q, 2}^{\dagger} \hat{\tilde{c}}_{p, 2}\right) \hat{n}_{1} \hat{n}_{2}\right] \text {, }} \\
& \sum_{\nu= \pm} \frac{\left\langle 0,-\left|\hat{P}_{-}^{0,0} \hat{\mathcal{H}}_{T} \hat{P}_{\nu}^{0,1} \hat{\mathcal{H}}_{T} \hat{P}_{-}^{0,0}\right| 0,-\right\rangle}{E_{-}^{0,0}-E_{\nu}^{0,1}}+\frac{\left\langle 2,-\left|\hat{P}_{-}^{1,1} \hat{\mathcal{H}}_{T} \hat{P}_{\nu}^{0,1} \hat{\mathcal{H}}_{T} \hat{P}_{-}^{1,1}\right| 2,-\right\rangle}{E_{-}^{0,0}-E_{\nu}^{0,1}}= \\
& \frac{2 t^{2}}{\Delta^{2}-\left(U+2 V_{z}-\Omega_{0}\right)^{2}}\left(\lambda-U-2 V_{z}+\frac{\Delta^{2}\left(\lambda+2 \Omega_{0}\right)}{\left(\Delta^{2}+\lambda^{2}+\lambda \Omega_{0}\right)}\right) \times \\
& {\left[\hat{\tilde{c}}_{q, 2}^{\dagger} \hat{\tilde{c}}_{p, 2}-\hat{\tilde{c}}_{q, 2}^{\dagger} \hat{\tilde{c}}_{p, 2} \hat{n}_{d}+\delta_{q, p} \hat{n}_{1} \hat{n}_{2}+\left(\hat{\tilde{c}}_{q, 2}^{\dagger} \hat{\tilde{c}}_{p, 2}-\hat{\tilde{c}}_{q, 1}^{\dagger} \hat{\tilde{c}}_{p, 1}\right) \hat{n}_{1} \hat{n}_{2}\right] \text {, }} \\
& \sum_{\nu= \pm} \frac{\left\langle 0,-\left|\hat{P}_{-}^{0,0} \hat{\mathcal{H}}_{T} \hat{P}_{\nu}^{0,1} \hat{\mathcal{H}}_{T} \hat{P}_{-}^{1,1}\right| 2,-\right\rangle}{E_{-}^{0,0}-E_{\nu}^{0,1}}+\frac{\left\langle 2,-\left|\hat{P}_{-}^{1,1} \hat{\mathcal{H}}_{T} \hat{P}_{\nu}^{0,1} \hat{\mathcal{H}}_{T} \hat{P}_{-}^{0,0}\right| 0,-\right\rangle}{E_{-}^{0,0}-E_{\nu}^{0,1}}= \\
& \frac{2 \Delta t^{2} / \Omega_{0}}{2 V_{z}-U+\frac{\lambda^{2}}{2 V_{z}-U+2 \Omega_{0}}} \sum_{i=1,2}\left[\hat{\tilde{c}}_{q, i}^{\dagger} \hat{\tilde{c}}_{p, \bar{i}}^{\dagger} \hat{d}_{\bar{i}} \hat{d}_{i}+\text { h.c. }\right] \text {, } \\
& \sum_{\nu= \pm} \frac{\left\langle 0,-\left|\hat{P}_{-}^{0,0} \hat{\mathcal{H}}_{T} \hat{P}_{\nu}^{1,0} \hat{\mathcal{H}}_{T} \hat{P}_{-}^{1,1}\right| 2,-\right\rangle}{E_{-}^{0,0}-E_{\nu}^{1,0}}+\frac{\left\langle 2,-\left|\hat{P}_{-}^{1,1} \hat{\mathcal{H}}_{T} \hat{P}_{\nu}^{1,0} \hat{\mathcal{H}}_{T} \hat{P}_{-}^{0,0}\right| 0,-\right\rangle}{E_{-}^{0,0}-E_{\nu}^{1,0}}= \\
& \frac{-2 \Delta t^{2} / \Omega_{0}}{2 V_{z}+U+\frac{\lambda^{2}}{2 V_{z}+U-2 \Omega_{0}}} \sum_{i=1,2}\left[\hat{\tilde{c}}_{q, i}^{\dagger} \hat{\tilde{c}}_{p, \bar{i}}^{\dagger} \hat{d}_{\bar{i}} \hat{d}_{i}+\text { h.c. }\right] \text {. }
\end{aligned}
$$


Now, by defining the iso-spin operators $\vec{I}_{d}$ of the DQD as

$$
\begin{gathered}
I_{d}^{x}=\frac{1}{2}\left(\hat{d}_{2}^{\dagger} \hat{d}_{1}^{\dagger}+\hat{d}_{1} \hat{d}_{2}\right), \\
I_{d}^{y}=\frac{i}{2}\left(\hat{d}_{2}^{\dagger} \hat{d}_{1}^{\dagger}-\hat{d}_{1} \hat{d}_{2}\right), \\
I_{d}^{z}=\frac{1}{2}\left(\hat{n}_{d}-1\right),
\end{gathered}
$$

with a similar definition for the $\vec{I}_{c}$ as the iso-spin operators of the electrodes and using the identities $2 \hat{n}_{1} \hat{n}_{2}=\hat{n}_{d}$ and $\hat{n}_{1}=\hat{n}_{2}$ which are valid in the ground state of the negative $U_{\text {eff }}$ regime, we can obtain the effective Hamiltonian in Eq. 16 .

1 A. C. Hewson, The Kondo problem to heavy fermions, vol. 2 (Cambridge university press, 1997).

2 T. W. Odom, J.-L. Huang, C. L. Cheung, and C. M. Lieber, Science 290, 1549 (2000).

3 M. R. Buitelaar, T. Nussbaumer, and C. Schönenberger, Phys. Rev. Lett. 89, 256801 (2002).

${ }^{4}$ L. H. Yu and D. Natelson, Nano Lett. 4, 79 (2004).

${ }^{5}$ R. Potok, I. Rau, H. Shtrikman, Y. Oreg, and D. Goldhaber-Gordon, Nature 446, 167 (2007).

6 Y.-S. Fu, S.-H. Ji, X. Chen, X.-C. Ma, R. Wu, C.-C. Wang, W.-H. Duan, X.-H. Qiu, B. Sun, P. Zhang, et al., Phys. Rev. Lett. 99, 256601 (2007).

7 M. R. Calvo, J. Fernandez-Rossier, J. J. Palacios, D. Jacob, D. Natelson, and C. Untiedt, Nature 458, 1150 (2009).

8 J. Pillet, C. Quay, P. Morfin, C. Bena, A. L. Yeyati, and P. Joyez, Nat. Phys. 6, 965 (2010).

9 E. J. Lee, X. Jiang, M. Houzet, R. Aguado, C. M. Lieber, and S. De Franceschi, Nat. Nanotech. 9, 79 (2014).

10 Z. Iftikhar, S. Jezouin, A. Anthore, U. Gennser, F. Parmentier, A. Cavanna, and F. Pierre, Nature 526, 233 (2015).

11 T. Inoshita, Science 281, 526 (1998).

12 S. M. Cronenwett, T. H. Oosterkamp, and L. P. Kouwenhoven, Science 281, 540 (1998).

13 M. Pustilnik and L. Glazman, J. Phys.: Condens. Matter 16, R513 (2004)

14 P. Jarillo-Herrero, J. Kong, H. S. Van Der Zant, C. Dekker, L. P. Kouwenhoven, and S. De Franceschi, Nature 434, 484 (2005).

15 S. Amasha, A. J. Keller, I. G. Rau, A. Carmi, J. A. Katine, H. Shtrikman, Y. Oreg, and D. Goldhaber-Gordon, Phys. Rev. Lett. 110, 046604 (2013).

16 Z.-q. Bao, A.-M. Guo, and Q.-f. Sun, J. Phys.: Condens. Matter 26, 435301 (2014).

17 A. Taraphder and P. Coleman, Phys. Rev. Lett. 66, 2814 (1991).

18 Y. Matsushita, H. Bluhm, T. H. Geballe, and I. R. Fisher, Phys. Rev. Lett. 94, 157002 (2005).

19 M. Dzero and J. Schmalian, Phys. Rev. Lett. 94, 157003 (2005).

20 M. Matusiak, E. M. Tunnicliffe, J. R. Cooper, Y. Matsushita, and I. R. Fisher, Phys. Rev. B 80, 220403 (2009).
21 T. A. Costi and V. Zlatić, Phys. Rev. Lett. 108, 036402 (2012).

22 G. Cheng, M. Tomczyk, S. Lu, J. P. Veazey, M. Huang, P. Irvin, S. Ryu, H. Lee, C.-B. Eom, C. S. Hellberg, et al., Nature 521, 196 (2015).

${ }^{23}$ G. E. Prawiroatmodjo, M. Leijnse, F. Trier, Y. Chen, D. V. Christensen, M. Soosten, N. Pryds, and T. S. Jespersen, Nat Commun. 8, 395 (2017).

24 T.-F. Fang, A.-M. Guo, H.-T. Lu, H.-G. Luo, and Q.-F. Sun, Phys. Rev. B 96, 085131 (2017).

25 L. Borda, G. Zaránd, W. Hofstetter, B. I. Halperin, and J. von Delft, Phys. Rev. Lett. 90, 026602 (2003).

26 A. S. Alexandrov, A. M. Bratkovsky, and R. S. Williams, Phys. Rev. B 67, 075301 (2003).

27 P. S. Cornaglia, H. Ness, and D. R. Grempel, Phys. Rev. Lett. 93, 147201 (2004).

28 M. R. Galpin, D. E. Logan, and H. R. Krishnamurthy, Phys. Rev. Lett. 94, 186406 (2005).

29 D. E. Liu, S. Chandrasekharan, and H. U. Baranger, Phys. Rev. Lett. 105, 256801 (2010).

30 I. Garate, Phys. Rev. B 84, 085121 (2011).

31 S. Andergassen, T. A. Costi, and V. Zlatić, Phys. Rev. B 84, 241107 (2011)

32 G. Yoo, J. Park, S.-S. B. Lee, and H.-S. Sim, Phys. Rev. Lett. 113, 236601 (2014).

33 G. Széchenyi, A. Pályi, and M. Droth, Phys. Rev. B 96, 245302 (2017).

34 A. Hamo, A. Benyamini, I. Shapir, I. Khivrich, J. Waissman, K. Kaasbjerg, Y. Oreg, F. von Oppen, and S. Ilani, Nature 535, 395 (2016).

35 W. Little, Phys. Rev. 134, A1416 (1964).

36 J. S. Lim, R. López, G. Platero, and P. Simon, Phys. Rev. B 81, 165107 (2010).

37 R. Bulla, T. A. Costi, and T. Pruschke, Rev. Mod. Phys. 80, 395 (2008).

${ }^{38} \mathrm{R}$. Zitko, The package is available at, URL http:// nrgljubljana.ijs.si/

${ }^{39}$ W. G. Van der Wiel, S. De Franceschi, J. M. Elzerman, T. Fujisawa, S. Tarucha, and L. P. Kouwenhoven, Rev. Mod. Phys. 75, 1 (2002). 